\title{
Adjunctive pharmacotherapy for cognitive deficits in schizophrenia: meta-analytical investigation of efficacy
}

Kee-Hong Choi, * Til Wykes and Matthew M. Kurtz*

\section{Background}

A growing number of studies have investigated the efficacy of novel, adjunctive pharmacotherapies for treatment of cognitive deficits in schizophrenia with conflicting results.

\section{Aims}

To investigate the comparative efficacy of these agents on cognition and symptoms in schizophrenia, and to identify promising cognitive domains and candidate medications that can be incorporated in treatment trials combined with cognitive remediation to maximise treatment effects.

\section{Method}

A total of 26 double-blind, placebo-controlled studies investigating medications targeted at cholinergic, glutamatergic or serotonergic receptor classes and with participants with schizophrenia or schizoaffective disorder were identified.

\section{Results}

Medications targeted at the cholinergic receptor class produced marginal improvements in verbal learning and memory $(d=0.23, P=0.06)$, and donepezil, a specific type of cholinergic agonist, produced a moderate effect $(d=0.58)$ on spatial learning and memory. Cholinergic and glutamatergic agents produced moderate effect-size improvements on negative symptoms ( $d=0.54$ and $d=0.62$ respectively), and small effect-size improvements on general symptoms ( $d=0.46$ and $d=0.41$ respectively). Serotonergic agents produced small effect-size improvements in positive symptoms $(d=0.33)$.

\section{Conclusions}

Cholinergic medications produced marginal improvement in verbal learning and memory and moderate improvements on spatial learning and memory, although there was no evidence to support the use of glutamatergic or serotonergic medications as a stand-alone treatment for improving cognitive function. Cholinergic and glutamatergic agents improved negative and general symptoms, whereas serotenergic medications improved positive symptoms.

\section{Declaration of interest}

None.
Abundant evidence indicates that deficits in cognition are a core feature of schizophrenia ${ }^{1}$ evident at illness onset, before antipsychotic treatment, ${ }^{2}$ that persist into senescence. ${ }^{3}$ Particular significance has been attached to these deficits as they account for the diversity of functional outcomes in the disorder more effectively than symptoms, and other illness features. ${ }^{4,5}$ Studies of first- and second-generation antipsychotic medications, although effective at diminishing positive symptoms, are largely neutral with respect to the cognitive features of the disorder. ${ }^{6,7}$ Thus, novel interventions are necessary to address socially disabling cognitive deficits. In recent years, a growing number of randomised controlled studies have revealed that behavioural-based training interventions designed to improve cognitive function, labelled cognitive remediation therapy, have shown durable effects on global cognition and functioning when administered as a component of other psychiatric rehabilitation. ${ }^{8}$ These results have promoted therapeutic optimism regarding the sensitivity of cognitive deficits in schizophrenia to treatment.

In parallel with behavioural treatments, a growing body of literature has evaluated the use of cognitive-enhancing medications prescribed as a supplement to primary antipsychotic pharmacotherapy to alter the functioning of neurotransmitter systems thought to underlie these cognitive deficits. Accordingly, medications that target several specific neurotransmitter receptor systems have been identified and tested: most commonly studied systems include the acetylcholinergic, glutamatergic and serotonergic systems. The rationale for the use of these agents has ranged from the putative role of the receptor class in the

*These authors contributed equally to this work. genesis of the disorder, receptors that enhance learning and memory in animal models and/or effects of these agents on cognition in other neuropsychiatric disorders without a specific link to schizophrenia. To date, three acetylcholinesterase inhibitors (AChEIs), which enhance synaptic transmission of acetylcholine (ACh), have been studied: donepezil, rivastigmine and galantamine. Findings from studies of adjunctive AChEI treatment have been mixed, with several studies, with donepezil and rivastigmine, some of large scale, showing no differences from placebo. ${ }^{9-13}$ In contrast, studies of galantamine, a more recently tested AChEI medication with additional positive allosteric modulatory effects at nicotinic $\alpha_{4} \beta_{2}$ and $\alpha_{7}$ receptors at lower doses, have offered more promise, with results showing improvement in verbal memory and processing speed ${ }^{14}$ or attention and delayed verbal and non-verbal memory. ${ }^{15-17}$

In recent years, studies have also investigated the effects of medications that enhance glutamate transmission by either glycine partial agonist actions (e.g. D-cycloserine), glycine full agonist actions (e.g. D-serine, D-alanine) or by inhibiting re-uptake of glycine (sarcosine) for enhancing $N$-methyl-D-aspartate (NMDA) channel opening through the glycine recognition site. Some studies have reported therapeutic gains for negative ${ }^{18}$ and overall psychiatric symptoms ${ }^{19}$ and executive function. ${ }^{20}$ Although largescale, multisite studies of partial glutamate agonists have been negative (i.e. D-cycloserine), ${ }^{21}$ positive findings on symptoms and cognition have been reported for D-serine, ${ }^{20,22}$ D-alanine ${ }^{23}$ and sarcosine $e^{24}$ in smaller, randomised trials.

Finally, investigators have studied medications that selectively enhance serotonin transmission $\left(5-\mathrm{HT}_{1 \mathrm{~A}}\right.$ agonists such as tandospirone and mianserin). ${ }^{25-28}$ These studies have yielded 
largely positive findings, with effects on learning and memory, ${ }^{26,28}$ executive-function ${ }^{26}$ and processing speed, ${ }^{25}$ but samples in these studies have been typically small and some negative findings have been reported. ${ }^{27}$

In sum, there are varied results from medication trials targeting acetylcholinergic, glutamatergic and serotonergic neurotransmitter systems that could be linked to the type of medication used to influence the targeted neurotransmitter system (i.e. donepezil and rivastigmine $v$. galantamine for AChEI medications; D-cycloserine $v$. D-serine, D-alanine and sarcosine for glutamatergic enhancing medications), small sample size, limited power and varied trial results within other classes of medications (i.e. $5-\mathrm{HT}_{1 \mathrm{~A}}$ agonists). A meta-analysis of these studies will permit pooling of results to assess effects across larger samples allowing comparisons between medication type within major medication classes (acetylcholine and glutamate) and the overall effects of different classes of medications where study sample sizes have been small and findings inconsistent (5- $\mathrm{HT}_{1 \mathrm{~A}}$ agonists). Another important impetus for this meta-analysis is to identify promising target domains of cognition and candidate medications that can be incorporated in treatment trials combined with cognitive remediation to maximise treatment effects. Based on putative mechanisms of drug action, we hypothesised that medications targeted at the cholinergic system would produce moderate effect-size improvements in overall cognitive function as well as learning and memory, with larger effects with the use of galantamine. We predicted non-significant effects of glutamatergic partial agonist medication on overall cognitive function, but small-to-moderate effects of other glutamatergic agents on overall cognitive function as well as measures of attention and learning and memory. Finally, we predicted small-to-moderate size effects of $5-\mathrm{HT}_{1 \mathrm{~A}}$ receptor agonists on overall cognitive function as well as attention, learning and memory and executive function. Given the link between cognitive deficits and negative symptoms, ${ }^{29}$ we predicted that improvements in cognition would be associated with reductions in negative but not positive symptoms.

\section{Method}

We followed the Quality of Reporting of Meta-analyses $(\text { QUOROM })^{30}$ methods for extraction of relevant studies and effect sizes.

\section{Search strategy}

Articles included in the meta-analysis were identified through a computer-based search of PubMed, MEDLINE and PsycINFO up to October 2011. The following search terms were used as keywords: ("cognitive enhancer" or "cognitive enhanc*) OR ("tacrine," "cholinesterase," "donepezil," "galantamine," "physostigmine," or "rivastigmine") OR ("glutamatergic," "glycine," "D-serine," "sarcosine," "D-alanine," "D-cycloserine:", "CX516”) OR ("serotonergic," "buspirone," "citalopram," "tropisetron," or "tandospirone") AND ("controlled trial") AND ("schizophrenia," "schizoaffective," or "schizo*"). The reference sections of articles located from all searches were studied for relevant citations.

\section{Inclusion criteria}

Ninety-three reports were examined according to the following criteria: (a) sample comprised exclusively of participants with a diagnosis of schizophrenia and/or schizoaffective disorder, (b) randomised or sample-matched, controlled trial that included a separate placebo parallel control group, (c) published in the English language, (d) included at least one standardised cognitive measure, (e) provided sufficient statistical detail to compute a $d$-value either from the published paper or a reply to a request for raw data, and (f) the studied agent targeted one of three broad receptor classes: cholinergic, glutamatergic or serotonergic systems. These criteria excluded 67 reports (criterion: (a) $n=2$; (b) $n=28$; (c) $n=1$; (d) $n=14$; (e) $n=2$ and (f) $n=20$ ). These procedures resulted in a final sample of 26 studies.

\section{Medication type}

Studies were grouped by mechanism of action, for example, the neurotransmitter system these medication types influenced most strongly: cholinergic agonists (i.e. donepezil, galantamine and rivastigmine), glutamate agonists (i.e. D-cycloserine, D-serine, CX516) or serotonergic agonists (i.e. buspirone, tandospirone, tropisetron and mianserin). These groupings are consistent with contemporary understanding of the pharmacological actions of these medications. ${ }^{31}$

\section{Study outcome measures}

In order to reduce the number of statistical comparisons and associated elevation of type I error, individual measures were grouped into eight cognitive domains and three psychiatric symptom domains (see online Table DS1). We selected Measurement and Treatment Research to Improve Cognition in Schizophrenia (MATRICS) categories, ${ }^{32}$ previous meta-analyses ${ }^{8,33}$ as well as the Gladsjo et $a l^{34}$ confirmatory factor analysis as guides for grouping cognitive measures in the current study. Where individual measures were different from those of MATRICS, McGurk et al's, ${ }^{33}$ Wykes et al's ${ }^{8}$ or Gladsjo et al's, ${ }^{34}$ measures were grouped according to the theoretical cognitive construct they were presumed to measure. Cognition composite scores were derived from studies when they were available. In instances in which a composite score was not presented, we created an overall composite effect size by averaging the available effect sizes of reported cognitive domains in the study. We excluded outcome measures: (a) that combined multiple cognitive domains in the same score (verbal and visual memory for example), or (b) did not fall into our cognitive or symptom or functioning domains (for example olfactory tests).

\section{Protecting against bias}

All study characteristics were coded independently by two raters (K-H.C. and M.M.K.) in a subsample of $20 \%$ of studies to ensure reliability of extraction of study characteristics, with high reliability $(95 \%)$.

\section{Meta-analytic procedure: calculation of effect sizes}

The unit of analysis in a meta-analysis is the effect size $(d)$. For purposes of the present study the $d$ score was defined as the difference between intervention type (i.e. treatment $v$. control) at termination of treatment expressed in standard deviation units $\left(\right.$ Mean $_{\text {post exp }}-$ Mean $_{\text {post control }} /$ s.d. .pooled across groups $_{\text {. Study }}$ statistics were converted to $d$ using formulas provided by Glass. ${ }^{35}$ We used the pooled standard deviation using the formula of Rosenthal. ${ }^{36}$ Because of the potential for inflated within-group effects relative to between-group comparisons, ${ }^{37}$ we did not compare within-group pre- to post-treatment change. Effect size for each cognitive domain was calculated in the following manner: to maximise the likelihood of detecting medication effects, for the first step, if there was a measure (for example the Wisconsin Card Sorting Test (WCST)) that had multiple outcome indices (for example categories, perseverative errors), we selected 
the outcome measure with the largest effect size to sensitively identify the most promising medications. For the second step, if there were multiple measures of a specific domain (such as Letter-Number Sequencing and digits backward for verbal working memory), we selected the middle effect-size value if there were an odd number of tests, and the higher of the two middlemost scores if there were an even number of tests in a domain. For studies that included multiple doses of adjunctive cognitive-enhancing medication, we selected the dose with the largest effect on overall cognition. Non-significant results lacking supporting statistical information were coded as an effect size of zero. ${ }^{38}$ By expressing effect size in standard deviation units, we were able to make a direct comparison of outcomes across studies. Effects were categorised as small $(d=0.2-0.4)$, moderate $(d=0.5-0.8)$ or large $\left(d>0.8\right.$ or greater). ${ }^{38}$ All effect sizes were expressed in a way such that positive values indicated improvement as a result of cognitive-enhancing medication.

\section{Statistical analysis}

For meta-analyses, we used a mixed-effects model. ${ }^{37}$ This procedure used a set of SPSS Version 18.0 macros (in Windows) developed by Lipsey \& Wilson ${ }^{37}$ for the overall analysis, the categorical moderator analysis and the weighted regression analysis. The random-effects variance component was based on the method of moments estimation. ${ }^{39}$ Based on raw means and standard deviations, $t, F$, or $P$ statistics reported in the individual study, ${ }^{36,40}$ an unbiased measure of effect size, Cohen's $d^{40,41}$ was calculated, combined across studies and weighted according to their variance. Potential differences in effect size between studies were analysed using the method of Hedges \& Olkin. ${ }^{40}$

To help address the 'file-drawer' problem (i.e. selective publication of positive results) we calculated a fail-safe $N$ for each class of outcome variable ${ }^{42}$ to estimate the number of studies that would be needed to render any observed effect size non-significant. In the absence of a universally accepted significance level for effect sizes, an effect size of 0.10 was considered non-significant. ${ }^{42}$

\section{Moderator variable analysis}

Sample characteristics of age, gender, ethnicity, duration of illness, education, smoker ratio, baseline cognitive functioning or psychiatric symptoms, study characteristics of type of cognitive-enhancing medication selected, duration of illness, weeks of stabilisation on antipsychotics, adjunct antipsychotic medication(s), funding source(s) and study region(s), respectively, were coded as potential moderators of effect size to test whether significant heterogeneity in effect sizes was evident.

Continuous data (such as age, duration of illness, and weeks of stabilisation on antipsychotic medication prior to adjunctive medication) were analysed with a continuous model, ${ }^{43}$ with a $z$-test for significance of model fit. Group comparisons were made for categorical moderator variables (for example types of medication). We used an alpha level of 0.05 and all statistical tests were two-tailed.

\section{Results}

\section{Study characteristics}

A total of 26 studies involving 1104 participants (550 for treatment and 554 for placebo control) were included in the analysis (Table 1 and online Tables DS2 and DS3). ${ }^{9-14,16,17,20-22,25-28,44-54}$ Fifteen studies (57.7\%) investigated cognitive-enhancing medication as an adjunct to treatment with an 'atypical' antipsychotic medication only, six studies $(23.1 \%)$ investigated cognitiveenhancing medication as an adjunct to treatment with conventional 'typical' antipsychotic medication only and five studies (19.2\%) studied cognitive-enhancing medication as an adjunct to treatment with either atypical or conventional antipsychotic medication. Total duration of clinical trials ranged from 4 to 24 weeks, with an average of 10.77 weeks (s.d.=6.12) without a significant difference in trial length between drug classes. The average weeks for stabilisation on antipsychotic medication before entering a clinical trial was 11.60 weeks (s.d. $=7.18)$. In terms of types of cognitive-enhancing medication, 13 studies (50.0\%) utilised an AChEI (i.e. donepezil, galantamine and rivastigmine), 7 studies $(26.9 \%)$ utilised a glutamate agonist (i.e. D-cycloserine, D-serine and CX516), and 6 studies (23.1\%) utilised a serotonergic drug (i.e. buspirone, tandospirone, tropisetron and mianserin).

\section{Effects of cognitive-enhancing medication on cognitive outcomes}

As shown in Table 2, AChEIs (donepezil, galantamine and rivastigmine) produced a favourable response on verbal learning and memory measures of borderline significance $(d=0.23$, $P=0.06,95 \%$ CI -0.01 to 0.46$)$. In addition, heterogeneity measures comparing each type of cognitive-enhancing medication within the class indicated that the weighted mean effect of AChEIs on spatial learning and memory was not stable, $Q_{W}(4)=10.45$, $P<0.05$. Moderator analyses comparing galantamine and donepezil (not rivastigmine because of a small sample size of less than three studies) indicated that donepezil had a moderate effect ( $d=0.58,95 \%$ CI $0.07-1.09$ ) on spatial learning and memory. The weighted mean effect sizes for other classes of medications were neither significantly different from zero nor heterogeneous.

\section{Effects of cognitive-enhancing medication on symptoms}

As shown in Table 2, AChEIs (donepezil, galantamine and rivastigmine) and glutamate agonists (i.e. D-cycloserine, D-serine, CX516) produced small effect-size improvement in measures of

\begin{tabular}{|c|c|c|}
\hline Variable & Treatment group & Control group \\
\hline \multicolumn{3}{|l|}{ Sample size } \\
\hline Mean (s.d.) & 21.15 (23.19) & $21.31(24.00)$ \\
\hline$\%$ reporting & 100.0 & 100.0 \\
\hline \multicolumn{3}{|l|}{ Age, years } \\
\hline Mean (s.d.) & $40.95(6.65)$ & $40.78(6.54)$ \\
\hline$\%$ reporting & 96.2 & 96.2 \\
\hline \multicolumn{3}{|l|}{ Male, \% } \\
\hline Mean (s.d.) & $65.52(15.74)$ & $70.93(16.55)$ \\
\hline$\%$ reporting & 84.6 & 84.6 \\
\hline \multicolumn{3}{|l|}{ White, \% } \\
\hline Mean (s.d.) & $48.06(16.58)$ & $53.53(29.25)$ \\
\hline$\%$ reporting & 46.2 & 46.2 \\
\hline \multicolumn{3}{|l|}{ Smokers, \% } \\
\hline Mean (s.d.) & $54.13(24.69)$ & $56.37(26.29)$ \\
\hline$\%$ reporting & 38.5 & 34.6 \\
\hline \multicolumn{3}{|l|}{ Education, years } \\
\hline Mean (s.d.) & $11.30(1.58)$ & $11.18(1.82)$ \\
\hline$\%$ reporting & 53.9 & 53.9 \\
\hline \multicolumn{3}{|c|}{ Illness duration, months } \\
\hline Mean (s.d.) & $79.67(96.37)$ & $80.74(96.26)$ \\
\hline$\%$ reporting & 69.2 & 69.2 \\
\hline \multicolumn{3}{|c|}{ Age at onset, years } \\
\hline Mean (s.d.) & $20.74(6.93)$ & $21.68(7.48)$ \\
\hline \% reporting & 34.6 & 34.6 \\
\hline \multicolumn{3}{|c|}{ Clinical Global Impression score } \\
\hline Mean (s.d.) & $3.56(0.56)$ & $3.65(0.49)$ \\
\hline$\%$ reporting & 26.9 & 26.9 \\
\hline
\end{tabular}


Table 2 Mean effect sizes for controlled studies of each type of adjunctive pharmacotherapy for schizophrenia organised by measured area of outcome

\begin{tabular}{|c|c|c|c|c|c|c|c|c|c|c|}
\hline & \multirow{2}{*}{$\begin{array}{l}\text { Studies } \\
n\end{array}$} & \multicolumn{3}{|c|}{ Effect size } & \multicolumn{2}{|c|}{ z statistic ${ }^{a}$} & \multicolumn{3}{|c|}{$Q_{w}$ statistic $^{b}$} & \multirow{2}{*}{$\begin{array}{l}\text { Fail-safe } \\
\qquad N\end{array}$} \\
\hline & & Effect size & s.e. & $95 \% \mathrm{Cl}$ & $z$ & $P$ & $Q_{w}$ & d.f. & $P$ & \\
\hline \multicolumn{11}{|l|}{ Acetylcholinesterase inhibitors } \\
\hline \multicolumn{11}{|l|}{ Cognition } \\
\hline Overall neurocognitive function & 13 & 0.05 & 0.11 & -0.16 to 0.27 & 0.48 & 0.630 & 4.63 & 12 & 0.97 & $\mathrm{n} / \mathrm{a}$ \\
\hline Attention/vigilance & 11 & -0.12 & 0.11 & -0.35 to 0.10 & -1.07 & 0.284 & 8.87 & 10 & 0.54 & $\mathrm{n} / \mathrm{a}$ \\
\hline Verbal learning and memory & 9 & 0.23 & 0.12 & -0.01 to 0.46 & 1.86 & 0.062 & 6.85 & 8 & 0.55 & 11 \\
\hline Verbal working memory & 9 & -0.08 & 0.12 & -0.32 to 0.16 & -0.65 & 0.517 & 12.02 & 8 & 0.15 & $\mathrm{n} / \mathrm{a}$ \\
\hline Spatial learning and memory & 5 & 0.21 & 0.18 & -0.15 to 0.57 & 1.14 & 0.253 & 10.45 & 4 & 0.03 & 6 \\
\hline Spatial working memory & 3 & 0.16 & 0.18 & -0.19 to 0.50 & 0.89 & 0.371 & 0.97 & 2 & 0.62 & 2 \\
\hline Reasoning and problem-solving & 8 & -0.11 & 0.14 & -0.38 to 0.16 & -0.81 & 0.418 & 4.46 & 7 & 0.73 & $\mathrm{n} / \mathrm{a}$ \\
\hline Speed of processing & 8 & 0.06 & 0.13 & -0.19 to 0.31 & 0.44 & 0.659 & 10.32 & 7 & 0.17 & $\mathrm{n} / \mathrm{a}$ \\
\hline \multicolumn{11}{|l|}{ Psychiatric symptoms } \\
\hline Overall psychiatric symptoms & 5 & 0.46 & 0.21 & 0.04 to 0.88 & 2.15 & 0.032 & 3.40 & 4 & 0.49 & 18 \\
\hline Positive symptoms & 5 & 0.01 & 0.22 & -0.42 to 0.44 & 0.05 & 0.961 & 0.84 & 4 & 0.93 & $\mathrm{n} / \mathrm{a}$ \\
\hline Negative symptoms & 5 & 0.54 & 0.22 & 0.10 to 0.98 & 2.41 & 0.016 & 5.82 & 4 & 0.21 & 22 \\
\hline \multicolumn{11}{|l|}{ Glutamate agonists } \\
\hline \multicolumn{11}{|l|}{ Cognition } \\
\hline Overall neurocognitive function & 7 & 0.06 & 0.15 & -0.22 to 0.35 & 0.44 & 0.661 & 3.65 & 6 & 0.72 & $\mathrm{n} / \mathrm{a}$ \\
\hline Attention/vigilance & 3 & -0.01 & 0.19 & -0.37 to 0.36 & -0.04 & 0.970 & 0.13 & 2 & 0.94 & $\mathrm{n} / \mathrm{a}$ \\
\hline Verbal learning and memory & 3 & 0.07 & 0.20 & -0.31 to 0.46 & 0.38 & 0.708 & 5.93 & 2 & 0.05 & $\mathrm{n} / \mathrm{a}$ \\
\hline Spatial learning and memory & 3 & 0.04 & 0.20 & -0.35 to 0.43 & 0.20 & 0.841 & 2.51 & 2 & 0.28 & $\mathrm{n} / \mathrm{a}$ \\
\hline Reasoning and problem-solving & 6 & -0.13 & 0.16 & -0.43 to 0.18 & -0.82 & 0.411 & 3.26 & 5 & 0.66 & $\mathrm{n} / \mathrm{a}$ \\
\hline Speed of processing & 4 & -0.03 & 0.18 & -0.38 to 0.32 & -0.17 & 0.862 & 2.25 & 3 & 0.52 & $\mathrm{n} / \mathrm{a}$ \\
\hline \multicolumn{11}{|l|}{ Psychiatric symptoms } \\
\hline Overall psychiatric symptoms & 4 & 0.41 & 0.20 & 0.01 to 0.81 & 2.02 & 0.044 & 11.09 & 3 & 0.01 & 13 \\
\hline Positive symptoms & 6 & 0.08 & 0.16 & -0.24 to 0.39 & 0.47 & 0.637 & 0.48 & 5 & 0.99 & $\mathrm{n} / \mathrm{a}$ \\
\hline Negative symptoms & 7 & 0.62 & 0.14 & 0.34 to 0.90 & 4.35 & 0.000 & 9.13 & 6 & 0.17 & 36 \\
\hline \multicolumn{11}{|l|}{$\begin{array}{l}5-H T_{1 A} \text { agonists } \\
\text { Cognition }\end{array}$} \\
\hline Overall neurocognitive function & 6 & 0.07 & 0.15 & -0.22 to 0.37 & 0.49 & 0.625 & 2.73 & 5 & 0.74 & $\mathrm{n} / \mathrm{a}$ \\
\hline Verbal learning and memory & 4 & 0.14 & 0.18 & -0.22 to 0.49 & 0.75 & 0.455 & 2.54 & 3 & 0.47 & 1 \\
\hline Reasoning and problem-solving & 5 & 0.09 & 0.16 & -0.22 to 0.41 & 0.58 & 0.565 & 2.11 & 4 & 0.72 & $\mathrm{n} / \mathrm{a}$ \\
\hline \multicolumn{11}{|l|}{ Psychiatric symptoms } \\
\hline Overall psychiatric symptoms & 5 & 0.12 & 0.17 & -0.21 to 0.44 & 0.70 & 0.484 & 0.90 & 4 & 0.92 & 1 \\
\hline Positive symptoms & 5 & 0.33 & 0.17 & 0.00 to 0.66 & 1.97 & 0.048 & 0.17 & 4 & 1.00 & 12 \\
\hline Negative symptoms & 4 & -0.31 & 0.22 & -0.74 to 0.11 & -1.45 & 0.148 & 1.38 & 3 & 0.71 & $\mathrm{n} / \mathrm{a}$ \\
\hline $\begin{array}{l}\text { n/a, not applicable. } \\
\text { a. Significance test within the group. } \\
\text { b. Homogeneity statistic. }\end{array}$ & & & & & & & & & & \\
\hline
\end{tabular}

overall psychiatric symptoms $(d=0.46,95 \%$ CI $0.04-0.88$ for AChEIs; $d=0.41,95 \%$ CI $0.01-0.81$ for glutamate agonists) and moderate effect-size improvement in measures of negative symptoms $(d=0.54,95 \%$ CI $0.10-0.98$ for AChEIs; $d=0.62$, 95\% CI $0.34-0.90$ for glutamine agonists). In addition, heterogeneity measures within each type of cognitive-enhancing medication indicated that the weighted mean effect of glutamate partial and full agonists on overall psychiatric symptoms was not stable, $Q_{\mathrm{W}}(3)=11.09, P<0.01$. Post hoc analyses indicated that D-serine produced large effect-size improvement in measures of overall psychiatric symptoms when added to non-clozapine antipsychotics, whereas CX516 produced large effect-size improvement in measures of overall psychiatric symptoms when added to clozapine. The $5-\mathrm{HT}_{1 \mathrm{~A}}$ agonists (i.e. buspirone, tandospirone, tropisetron, mianserin) produced a small effect-size improvement in measures of positive symptoms ( $d=0.33,95 \%$ CI $0.00-0.66)$, but no effects for negative symptoms or overall psychiatric symptoms (Fig. 1).

\section{Discussion}

\section{Main findings}

This is the first study, to our knowledge, to meta-analyse placebocontrolled studies of three of the most commonly studied cognitive-enhancing adjunctive medication classes (cholinergic, glutamatergic and serotonergic) in schizophrenia. A total of 26 studies met our inclusion criteria. Notably, the majority of studies in this area did not employ a randomised controlled trial design $(n=28)$ and thus were excluded. In support of our hypothesis we found marginal evidence that cholinergic-enhancing medications produced improvements in verbal learning and memory $(d=0.23, P=0.06)$ relative to a placebo control in double-blind study designs. Also consistent with our hypotheses, two of three medication classes - cholinergic and glutamatergic drugs produced a moderate effect-size reduction in negative symptoms ( $d=0.54$ and 0.62 respectively) with no effect on positive symptoms. Inconsistent with our hypotheses we failed to find: (a) an effect of cholinergic, glutamatergic and serotonergic drugs, or any specific types of medications within these drug classes, on composite measures of cognition, or (b) an effect of glutamate or serotonergic drugs on any of the specific MATRICS-defined domains of cognition, attention/vigilance, verbal learning and memory, verbal working memory, spatial learning and memory, spatial working memory, reasoning and problem-solving or speed of processing. These findings are largely congruent with recent literature reviews in this research area, ${ }^{31,55}$ but shed additional light on this issue by providing a quantitative estimate of effects across studies. We note in the results of this meta-analysis that 


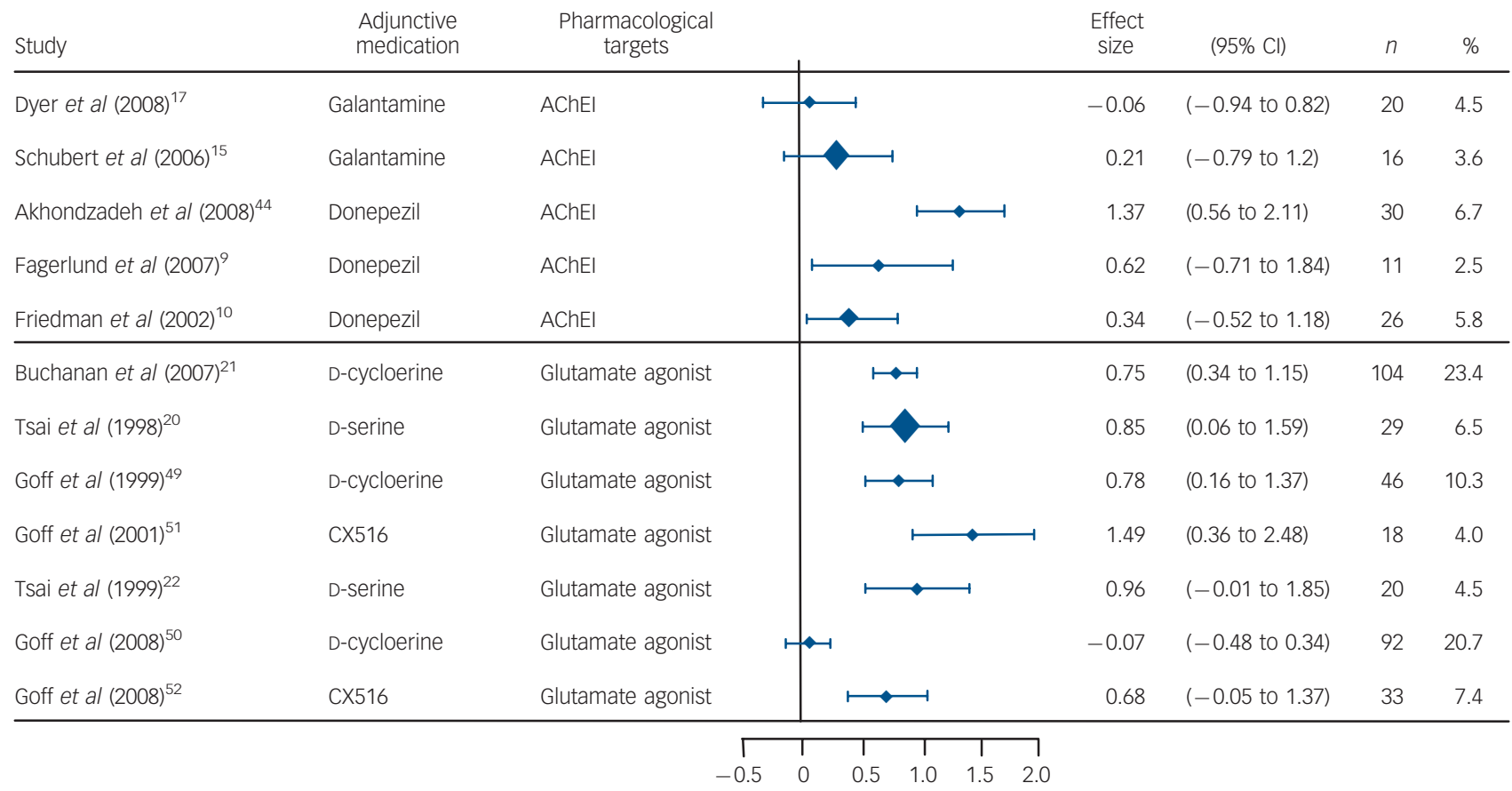

Fig. 1 Forest plot of negative symptom improvement among placebo-controlled studies of cholinergic and glutamatergic medications for cognitive enhancement in schizophrenia.

AChEl, acetylcholinesterase inhibitor.

cholinergic and glutamatergic add-on agents also produced small effect-size improvements on overall psychiatric symptoms, whereas serotonergic add-on agents produced small effect-size improvements on positive symptoms.

\section{Possible explanations for our findings}

There are many factors beyond the lack of active effects of adjunctive medication on cognitive outcomes that may have influenced the observed findings. First, timed cognitive tests that depend on performance speed for success may not be the most effective means for assessing changes in cognition that are represented in improved accuracy. For example, some have speculated that untimed neuropsychological tests, which have not typically been incorporated in standard neuropsychological assessment batteries, might capture functionally significant improvement in cognitive style such as taking time to plan a task or group information, which could be linked to aspects of social behaviours and negative symptoms. Thus, at least some of the reported improvements in negative symptoms in the current paper could have been related to improvements in cognition that were not effectively assessed by selected cognitive batteries. ${ }^{56}$

Second, although the MATRICS battery has shown minimal evidence of practice effects in drug treatment trials, ${ }^{57}$ many of the studies in this meta-analysis included measures that may be more susceptible to these effects. Although research in schizophrenia has shown only modest practice effects on the many commonly used cognitive tests, ${ }^{58,59}$ at least one study in this review showed improvement on the WCST in their control group that they attributed to practice effects, ${ }^{13}$ and verbal memory measures, without use of alternate forms, show moderate practice effects in schizophrenia. ${ }^{60}$ This concern is magnified by some indications that practice effects may be larger in trials of cognitive-enhancing medications, where there is an expectation of improved cognitive function. ${ }^{11}$ The high placebo response rate is particularly salient in psychiatric medication trials and also may have obscured potential drug benefit. Arguing against this possibility, there was evidence in our data of a correlation between larger drug effects on cognition in trials with shorter test-retest intervals (when practice effects ostensibly would be largest), but findings regarding this relationship were based on just three studies and non-significant.

Third, less than half of the studies included in this metaanalysis $(n=10)$ included a measure for adherence, thus it remains unknown whether weak effects could relate to this factor.

Nonetheless, findings to date indicate that pharmacotherapy targeted at specific neurotransmitter systems or receptor classes thought to underlie the cognitive impairment in schizophrenia has yielded only modest effects on specific domains of cognitive skill. These findings might suggest that the cognitive impairment evident in schizophrenia is reflective of more systemic deficits, generalised grey and white matter abnormalities or poor signal integration across a variety of neural systems indicative of a generalised neurodevelopmental or neurodegenerative process. This suggestion is consistent with analyses of a substantial body of neuropsychological literature suggesting that cognition in schizophrenia is characterised by poor performance across a variety of cognitive domains, rather than a pattern of specific deficits in isolated cognitive domains linked to specialised neural systems. ${ }^{61}$ These findings might also suggest that pharmacotherapy focused on neuroprotection such as antioxidants or anti-inflammatory agents, ${ }^{62}$ or promoting neurogenesis, ${ }^{63}$ might be more likely to yield pro-cognitive effects in schizophrenia.

\section{Implications}

The results of this meta-analysis suggest that cholinergic enhancing medications that marginally improve verbal learning and memory as a stand-alone treatment would be most likely to 
maximise treatment effects when administered in concert with empirically supported cognitive remediation therapy for cognitive deficits in schizophrenia. ${ }^{8}$ Given the functional significance of verbal learning and memory in schizophrenia ${ }^{64}$ it should be examined whether the addition of add-on cholinergic-enhancing medications in cognitive remediation treatment trials could produce a more powerful effect on outcomes.

It is speculated that symptom improvements produced by cholinergic, glutamatergic or serotonergic receptor medications might be related to factors such as downstream effects of cognitive-enhancing medications at dopaminergic synapses, as well as subtle improvements in cognition that could influence the display of psychiatric symptoms. ${ }^{59}$ It is also speculated that observed improvements in negative symptoms not accompanied by improved cognition in trials of glutamatergic medications might also suggest that the neural mechanisms necessary for negative symptom reduction may be more circumscribed than those for cognitive improvement.

\section{Limitations}

Several caveats to the results of this meta-analysis should be mentioned. First, the number of randomised, placebo-controlled studies for many classes of cognitive-enhancing medications remain small (for example for $5-\mathrm{HT}_{1 \mathrm{~A}}$ agonists there were six studies). This limited our capacity to test multiple cognitive domains for some agent classes (for example two cognitive domains for $5-\mathrm{HT}_{1 \mathrm{~A}}$ agonists) as well as the impact of potential moderators that might be expected to show significant effects. Second, the number of studies for some outcome domains was small and results should be considered as preliminary. Third, in the analysis of effects of these interventions on overall cognition, when overall scores were not provided in the specific study, we averaged the individual domain scores within each study. As covariance was not accounted for in this averaging, these effect sizes may represent an underestimate of the true effect. ${ }^{65}$ A related concern is that effects of medications on specific cognitive skills assessed by individual tests could be obscured by averaging results from these tests into broader MATRICS domains. We note, however, that analysis of mean effect sizes from the five most frequently selected tests (Continuous Performance Task, WCST, Stroop, California Verbal Learning Test and Hopkins Verbal Learning Test) across studies failed to reveal treatment effects within any medication class. Fourth, summary statistics for the studies in this analysis (Table 1) suggest that the vast majority of participants were individuals whose condition was chronic, and thus it remains unclear whether adjunctive cognitiveenhancing medication may have effects on people with schizophrenia in earlier stages of illness.

In summary, the results of this meta-analysis revealed small effects of cholinergic adjunctive pharmacotherapies on verbal learning and memory deficits in schizophrenia, with no effects of any class of pharmacological agent studied in this meta-analysis (cholinergic, glutamatergic or serotonergic) on overall cognition. Cholinergic and glutamatergic agents produced small to moderate effect-size improvements in general and negative symptoms, whereas serotonergic agents produced small improvements in positive symptoms. With respect to cognitive outcomes, although confounding effects in these studies cannot be ruled out, these findings suggest that future research may be better targeted at the assessment of medications that provide broader neuroprotective effects across a variety of neural systems that might better account for the diffuse neural damage hypothesised to underlie the disorder, and that might also enhance the effects of other, empirically supported cognitive interventions.
Kee-Hong Choi, PhD, Department of Psychology, Korea University, Seoul, South Korea; Til Wykes, PhD, Institute of Psychiatry, Kings College London, UK; Matthew M. Kurtz, PhD, Department of Psychology and Program in Neuroscience and Behavior, Wesleyan University, Middletown, Connecticut, USA

Correspondence: Matthew M. Kurtz, PhD, Department of Psychology, Wesleyan University, Judd Hall, 207 High Street, Middletown, Connecticut 06459, USA. Email: mkurtz@wesleyan.edu

First received 8 Dec 2011, final revision 12 Feb 2013, accepted 3 Apr 2013

\section{Funding}

This work was supported in part by the National Institute of Mental Health (K08 MH-69888), and the National Alliance for Research in Schizophrenia and Depression (Young Investigator Award; M.K.K.)

\section{Acknowledgements}

We thank Drs Kenji Hashimoto (Chiba University, Chiba, Japan) and Tomiki Sumiyoshi (University of Toyama, Toyama, Japan) for giving us access to their data.

\section{References}

1 Green MF, Nuechterlein KH. Should schizophrenia be treated as a neurocognitive disorder? Schizophr Bull 1999; 25: 309-19.

2 Saykin AJ, Shtasel DL, Gur RE, Kester DB, Mozley LH, Stafiniak P, et al. Neuropsychological deficits in neuroleptic naive patients with first-episode schizophrenia. Arch Gen Psychiatry 1994; 51: 124-31.

3 Harvey P, Silverman J, Mohs R, Parrella M, White L, Powchik P, et al. Cognitive decline in late-life schizophrenia: a longitudinal study of geriatric chronically hospitalized patients. Biol Psychiatry 1999; 45: 32-40.

4 Green MF, Kern RS, Braff DL, Mintz J. Neurocognitive deficits and functional outcome in schizophrenia: are we measuring the "right stuff"? Schizophr Bull 2000; 26: 119-36.

5 Green MF. Cognitive impairment and functional outcome in schizophrenia and bipolar disorder. J Clin Psychiatry 2006; 67: 3-8.

6 Harvey PD, Keefe RSE. Studies of cognitive change in patients with schizophrenia following novel antipsychotic treatment. Am J Psychiatry 2001; 158: 176-84.

7 Keefe S, Bilder R, Davis S, Harvey P, Palmer B, Gold J, et al. Neurocognitive effects of antipsychotic medications in patients with chronic schizophrenia in the CATIE trial. Arch Gen Psychiatry 2007; 64: 633-47.

8 wykes T, Huddy V, Cellard C, McGurk SR, Czobor P. A meta-analysis of cognitive remediation for schizophrenia: methodology and effect sizes. Am J Psychiatry 2011; 168: 472-85

9 Fagerlund B, Søholm B, Fink-Jensen A, Lublin H, Glenthøj BY. Effects of donepezil adjunctive treatment to ziprasidone on cognitive deficits in schizophrenia: a double-blind, placebo-controlled study. Clin Neuropharmacol 2007; 30: 3-12.

10 Friedman Jl, Adler DN, Howanitz E, Harvey PD, Brenner G, Temporini $\mathrm{H}$, et al. A double blind placebo controlled trial of donepezil adjunctive treatment to risperidone for the cognitive impairment of schizophrenia. Biol Psychiatry 2002; 51: 349-57

11 Keefe RS, Malhotra AK, Meltzer HY, Kane JM, Buchanan RW, Murthy A, et al. Efficacy and safety of donepezil in patients with schizophrenia or schizoaffective disorder: significant placebo/practice effects in a 12-week, randomized, double-blind, placebo-controlled trial. Neuropsychopharmacol 2008; 33: 1217-28.

12 Kohler CG, Martin EA, Kujawski E, Bilker W, Gur RE, Gur RC. No effect of donepezil on neurocognition and social cognition in young persons with stable schizophrenia. Cogn Neuropsychiatry 2010; 12: 412-21.

13 Sharma T, Reed C, Aasen I, Kumari V. Cognitive effects of adjunctive 24-weeks Rivastigmine treatment to antipsychotics in schizophrenia: a randomized, placebo-controlled, double-blind investigation. Schizophr Res 2006; 85: 73-83.

14 Buchanan RW, Conley RR, Dickinson D, Ball MP, Feldman S, Gold JM, et al. Galantamine for the treatment of cognitive impairments in people with schizophrenia. Am J Psychiatry 2008; 165: 82-9.

15 Schubert MH, Young KA, Hicks PB. Galantamine improves cognition in schizophrenic patients stabilized on risperidone. Biol Psychiatry 2006; 60: 530-3.

16 Lee SW, Lee JG, Lee BJ, Kim YH. A 12-week, double-blind, placebo-controlled trial of galantamine adjunctive treatment to conventional antipsychotics 
for the cognitive impairments in chronic schizophrenia. Int Clin Psychopharmacol 2007; 22: 63-8.

17 Dyer MA, Freudenreich O, Culhane MA, Pachas GN, Deckersbach T, Murphy $\mathrm{E}$, et al. High-dose galantamine augmentation inferior to placebo on attention, inhibitory control and working memory performance in nonsmokers with schizophrenia. Schizophr Res 2008; 102: 88-95.

18 Goff DC, Tsai G, Manoach DS, Flood J, Darby DG, Coyle JT. D-cycloserine added to clozapine for patients with schizophrenia. Am J Psychiatry 1996; 153: $1628-30$.

19 Tsai G, Lane HY, Yang P, Chong MY, Lange N. Glycine transporter I inhibitor $\mathrm{N}$-methylglycine (sarcosine), added to antipsychotics for the treatment of schizophrenia. Biol Psychiatry 2004; 55: 452-56.

20 Tsai G, Yang P, Chung LC, Lange N, Coyle JT. D-serine added to antipsychotics for the treatment of schizophrenia. Biol Psychiatry 1998; 44: 1081-9.

21 Buchanan RW, Javitt DC, Marder SR, Schooler NR, Gold JM, McMahon RP, et al. The Cognitive and Negative Symptoms in Schizophrenia Trial (CONSIST) the efficacy of glutamatergic agents for negative symptoms and cognitive impairments. Am J Psychiatry 2007; 164: 1593-602.

22 Tsai GE, Yang P, Chung LC, Tsai I. D-serine added to clozapine for the treatment of schizophrenia. Am J Psychiatry 1999; 156: 1822-5.

23 Tsai GE, Yang P, Chang YC, Chong MY. D-alanine added to antipsychotics for the treatment of schizophrenia. Biol Psychiatry 2006; 59: 230-4.

24 Lane $\mathrm{HY}$, Liu $\mathrm{YC}$, Huang $\mathrm{CL}$, Chang $\mathrm{YC}$, Liau $\mathrm{CH}$, Perng $\mathrm{CH}$, et al. Sarcosine (N-methylglycine) treatment for acute schizophrenia: a randomized, double-blind study. Biol Psychiatry 2008; 63: 9-12.

25 Sumiyoshi T, Park S, Jayathilake K, Roy A, Ertugrul A, Meltzer HY. Effect of buspirone, a serotonin1A partial agonist, on cognitive function in schizophrenia: a randomized, double-blind, placebo-controlled study. Schizophr Res 2007; 95: 158-68.

26 Sumiyoshi T, Matsui M, Nohara S, Yamashita I, Kurachi M, Sumiyoshi C, et al. Enhancement of cognitive performance in schizophrenia by addition of tandospirone to neuroleptic treatment. Am J Psychiatry 2001; 158: 1722-5.

27 Pikuli D, Olver JS, Maruff $P$, Norman TR. Treatment of cognitive dysfunction in chronic schizophrenia by augmentation of atypical antipsychotics with buspirone, a partial 5 HT1A receptor agonist. Hum Psychopharm Clin 2009; 24: 437-46.

28 Poyurovsky M, Koren D, Gonopolsky I, Schneidman M, Fuchs C, Weizman A et al. Effect of the 5-HT2 antagonist mianserin on cognitive dysfunction in chronic schizophrenia patients: an add-on, double-blind placebo-controlled study. Eur Neuropsychopharmacol 2003; 13: 123-8.

29 de Gracia Dominguez M, Viechtbauer W, Simons CJP, van Os J, Krabbendam L. Are psychotic psychopathology and neurocognition orthogonal? A systematic review of their associations. Psychol Bull 2009; 135: $157-71$.

30 Moher D, Cook DJ, Eastwood S, Olkin I, Rennie D, Stroup DF. Improving the quality of reports of meta-analyses of randomised controlled trials: the QUOROM statement. Lancet 1999; 354: 1896-900.

31 Goff DC, Hill M, Barch D. The treatment of cognitive impairment in schizophrenia. Pharmacol Biochem Behav 2011; 99: 245-53.

32 Nuechterlein KH, Barch DM, Gold JM, Goldberg TE, Green MF, Heaton RK. Identification of separable cognitive factors in schizophrenia. Schizophr Res 2004; 72: 29-39.

33 McGurk SR, Twamley EW, Sitzer DI, McHugo GJ, Mueser KT. A meta-analysis of cognitive remediation in schizophrenia. Am J Psychiatry 2007; 164: 1791-802

34 Gladsjo JA, McAdams LA, Palmer BW, Moore DJ, Jeste DV, Heaton RK A six-factor model of cognition in schizophrenia and related psychotic disorders: relationships with clinical symptoms and functional capacity. Schizophr Bull 2004; 30: 739-54.

35 Glass GV. Integrating findings: the meta-analysis of research. Rev Res Educ 1977; 5: 351-79.

36 Rosenthal R. Parametric measures of effect size. In The Handbook of Research Synthesis (eds HM Cooper, LV Hedges): 231-44. Sage, 1994.

37 Lipsey MW, Wilson DB. The way in which intervention studies have "personality" and why it is important to meta-analysis. Eval Health Prof 2001; 24: 236-54.

38 Glass GV, McGaw B, Smith ML. Meta-analysis in Social Research. Sage, 1981

39 Hedges LV, Vevea JL. Fixed-and random-effects models in meta-analysis Psychol Methods 1998; 3: 486-504.

40 Hedges LV, Olkin I. Statistical Methods for Meta-Analysis. Academic Press, 1985.

41 Hedges LV. Distribution theory for Glass's estimator of effect size and related estimators. J Educ Stat 1981; 6: 107-28.
42 Orwin RG. A fail-safe $\mathrm{N}$ for effect size in meta-analysis. J Educ Stat 1983; 8 157-9.

43 Kohler CG, Walker JB, Martin EA, Healey KM, Moberg PJ. Facial emotion perception in schizophrenia: a meta-analytic review. Schizophr Bull 2010; 36: 1009-19.

44 Akhondzadeh S, Gerami M, Noroozian M, Karamghadiri N, Ghoreishi A Abbasi SH, et al. A 12-week, double-blind, placebo- controlled trial of donepezil adjunctive treatment to risperidone in chronic and stable schizophrenia. Prog Neuropsychopharmacol Biol Psychiatry 2008; 32 1810-5.

45 Freudenreich O, Herz L, Deckersbach T, Evins AE, Henderson DC, Cather C, et al. Added donepezil for stable schizophrenia: a double-blind, placebocontrolled trial. Psychopharmacology (Berl) 2005; 181: 358-63.

46 Lindenmayer JP, Khan A. Galantamine augmentation of long-acting injectable risperidone for cognitive impairments in chronic schizophrenia. Schizophr Res 2011; 125: 267-77.

47 Lee BJ, Lee JG, Kim YH. A 12-week, double-blind, placebo-controlled trial of donepezil as an adjunct to haloperidol for treating cognitive impairments in patients with chronic schizophrenia. J Psychopharmacol 2007; 21: 421-7

48 Schubert $\mathrm{MH}$, Young KA, Hicks PB. Galantamine improves cognition in schizophrenic patients stabilized on risperidone. Biol Psychiatry 2006; 60 530-3.

49 Goff DC, Tsai G, Levitt J, Amico E, Manoach D, Schoenfeld DA, et al. A placebo-controlled trial of D-cycloserine added to conventional neuroleptics in patients with schizophrenia. Arch Gen Psychiatry 1999; 56: $21-7$.

50 Goff DC, Cather C, Gottlieb JD, Evins AE, Walsh J, Raeke L, et al. Once-weekly D-cycloserine effects on negative symptoms and cognition in schizophrenia: an exploratory study. Schizophr Res 2008; 106: 320-7.

51 Goff DC, Leahy L, Berman I, Posever T, Herz L, Leon AC, et al. A placebo-controlled pilot study of the ampakine CX516 added to clozapine in schizophrenia. J Clin Psychopharmacol 2001; 21: 484-7.

52 Goff DC, Lamberti JS, Leon AC, Green MF, Miller AL, Patel J, et al. A placebo-controlled add-on trial of the Ampakine, CX516, for cognitive deficits in schizophrenia. Neuropsychopharmacol 2008; 33: 465-72.

53 Sumiyoshi T, Matsui M, Yamashita I, Nohara S, Kurachi M, Uehara T, et al. The effect of tandospirone, a serotonin (1A) agonist, on memory function in schizophrenia. Biol Psychiatry 2001; 49: 861-8.

54 Shiina A, Shirayama Y, Niitsu T, Hashimoto T, Yoshida T, Hasegawa T, et al. A randomised, double-blind, placebo-controlled trial of tropisetron in patients with schizophrenia. Ann Gen Psychiatry 2010; 9: 1-10.

55 Keefe RSE, Buchanan RW, Marder SR, Schooler NR, Dugar A, Zivkov M, et al. Clinical trials of potential cognitive-enhancing drugs in schizophrenia: what have we learned so far? Schizophr Bull 2013; 39: 417-35.

56 Wykes T, Spaulding WD. Thinking about the future cognitive remediation therapy - what works and could we do better? Schizophr Bull 2011; 37 (suppl 2): S80-90.

57 Keefe RSE, Fox KH, Harvey PD, Cucchiaro J, Siu C, Loebel A. Characteristics of the MATRICS Consensus Cognitive Battery in a 29-site antipsychotic schizophrenia clinical trial. Schizophr Res 2011; 125: 161-8.

58 Keefe RSE, Goldberg TE, Harvey PD, Gold JM, Poe MP, Coughenour L. The Brief Assessment of Cognition in Schizophrenia: reliability, sensitivity, and comparison with a standard neurocognitive battery. Schizophr Res 2004; 68: $283-97$

59 Nuechterlein KH, Green MF, Kern RS, Baade LE, Barch DM, Cohen JD, et al. The MATRICS Consensus Cognitive Battery, part 1: test selection, reliability, and validity. Am J Psychiatry 2008; 165: 203-13.

60 Hawkins KA, Wexler BE. California Verbal Learning Test practice effects in a schizophrenia sample. Schizophr Res 1999; 39: 73-8.

61 Dickinson D, Harvey PD. Systemic hypotheses for generalized cognitive deficits in schizophrenia: a new take on an old problem. Schizophr Bull 2009; 35: 403-14.

62 Amminger GP, Schäfer MR, Papageorgiou K, Klier CM, Cotton SM, Harrigan $\mathrm{SM}$, et al. Long-chain-3 fatty acids for indicated prevention of psychotic disorders. Arch Gen Psychiatry 2010; 67: 146-54.

63 Javitt DC. Effects of intranasal AL-108 (davunetide) on neurocognition and functional outcome in schizopherenia. Schizophr Res 2010; 117: 118-9.

64 Green MF. What are the functional consequences of neurocognitive deficits in schizophrenia? Am J Psychiatry 1996; 153: 321-30.

65 Gleser L, Olkin I. Stochastically dependent effect sizes. In The Handbook of Research Synthesis (eds HM Cooper, LV Hedges): 339-55. Sage Publications, 1994.

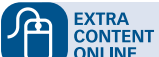

